\title{
Annually Recurring Erythema Annulare Centrifugum: A New Case Series with Review of the Literature
}

\author{
Martina Maurellia Paolo Gisondia Chiara Colato ${ }^{b}$ \\ Giampiero Girolomoni ${ }^{a}$ \\ aSection of Dermatology and Venereology, Department of Medicine, University of Verona, \\ Verona, Italy; bSection of Pathology, Department of Diagnostics and Public Health, \\ University of Verona, Verona, Italy
}

\section{Keywords}

Annually recurring erythema annulare centrifugum - Erythema annulare centrifugum .

Treatment

\begin{abstract}
Annually recurring erythema annulare centrifugum (AR-EAC) is a rare variant, characterized by typical annular plaques recurring in the same period of the year. We describe 5 new cases and present a review of the literature. Patients were 3 females and 2 males with an age range of 25-55 years. Multiple annular plaques were located at the thighs in 4 patients and the neck in one patient. In 1 patient, a single lesion was present. Plaques were recurring in summer in 3 cases; in 1 case, in spring; and another patient, in winter since 3-4 years. Lesions were self-healing in few days or weeks. Histologically, the epidermis presented mild acanthosis with patchy spongiosis, slight parakeratosis, and mild exocytosis. There was a perivascular lympho-histiocytic infiltrate of variable intensity in the superficial dermis, with occasional eosinophils. In 1 case, the inflammatory infiltrate reached the deep dermis. Mucin deposition was absent. Phenotyping studies in 1 case revealed a predominance of $T$ cells, with a small B-cell component. Moreover, a moderate number of CD123+ plasmacytoid dendritic cells and $\mathrm{CD} 1 \mathrm{a}^{+}$dendritic cells were noted. Fourteen cases of AR-EAC have been published previously. Collectively, patients' age ranged from 16 to 83 years, with a mean age of 47 years and a disease duration of 1-30 years. Lesions affected more frequently extremities and recurred most commonly in summer. Patients were all in good general health. Topical corticosteroids were the mainstay of treatment. AR-EAC is a benign disorder, the nature of which remains enigmatic.
\end{abstract}

\section{Karger $\stackrel{2}{=}$}


Maurelli et al.: Annually Recurring Erythema Annulare Centrifugum

\section{Introduction}

Annually recurring erythema annulare centrifugum (AR-EAC) is an EAC variant characterized by the appearance of annular plaques usually localized at the extremities recurring strikingly in the same period of the year. The lesions regress spontaneously after a variable period of days to few weeks. AR-EAC is apparently not associated with extracutaneous diseases, except in 2 cases affected by the hereditary lactate dehydrogenase M-subunit deficiency [1-3]. Here, we report 5 new cases and performed a narrative review of the published cases.

\section{Material and Methods}

Clinical records of patients affected by AR-EAC were retrieved from 2005 to 2020 by consulting the electronic medical records of the Section of Dermatology and Pathology of the University Hospital of Verona. Patients' information and images were analyzed, and data regarding age, gender, medical history, location, seasonal recurrence and duration of disease, clinical appearance of the lesions, symptoms, treatment details, and outcomes were collected. All the diagnoses were confirmed by histology. Representative paraffinembedded tissue sections were processed for immunohistochemical studies. A narrative literature review based on electronic search on PubMed ${ }^{\circledR}$ was performed. Original articles describing cases of AR-EAR published up to November 1, 2020 were retrieved. The key words used were "annually recurring erythema annulare centrifugum," "annually recurring acro-erythema," "annually recurring acroerythema," and "annually recurrent acroerythema." Two investigators (M.M. and C.C.) independently extracted data. A total of 12 articles were screened by title and abstract. All of them deemed relevant and were reviewed in full text.

\section{Results}

We report 5 new cases of AR-EAC. The first was a 55-year-old man presented to our attention with some erythematous and violaceous self-healing annular plaques at the thighs relapsing for 4 years at the end of summer. The second case was a 27-year-old woman complaining of spontaneously regressing annular plaque, located at the left tight, recurring for 3 consecutive years, always at the end of winter. The third patient was a 32-year-old woman presented with some very itchy self-healing annular plaques at the neck, presenting for 3 years always in spring (Fig. 1a, b). The fourth case was a 34-year-old woman affected with erythematous annular plaques, localized bilaterally at the thighs for 3 consecutive years in summer (Fig. 1c). The fifth case was a 25-year-old man complaining of some erythematous annular plaques at the thighs for 3 years, always in summer. In all 5 cases, the lesions presented with the same clinical characteristics, and the patients were all in good general healthy, without any comorbidities. No obvious trigger factors have been identified. The patients have been treated with topical corticosteroids and/or 2-week course of $0.5 \mathrm{mg} / \mathrm{kg}$ oral prednisone. Histopathology was very similar in all cases. The epidermis presented mild acanthosis with patchy spongiosis, slight parakeratosis, and mild exocytosis. An inflammatory perivascular lympho-histiocytic infiltrate of variable intensity was present in the papillary and mid-dermis, with occasional eosinophils. In patient 3 , the histology was characterized by a superficial and deep perivascular and periadnexal lymphohistiocytic infiltrate (Fig. 2). A few lymphocytes extended to follicular epithelium. Mucin deposition was absent. Phenotyping studies of

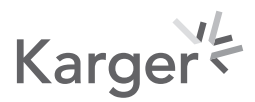




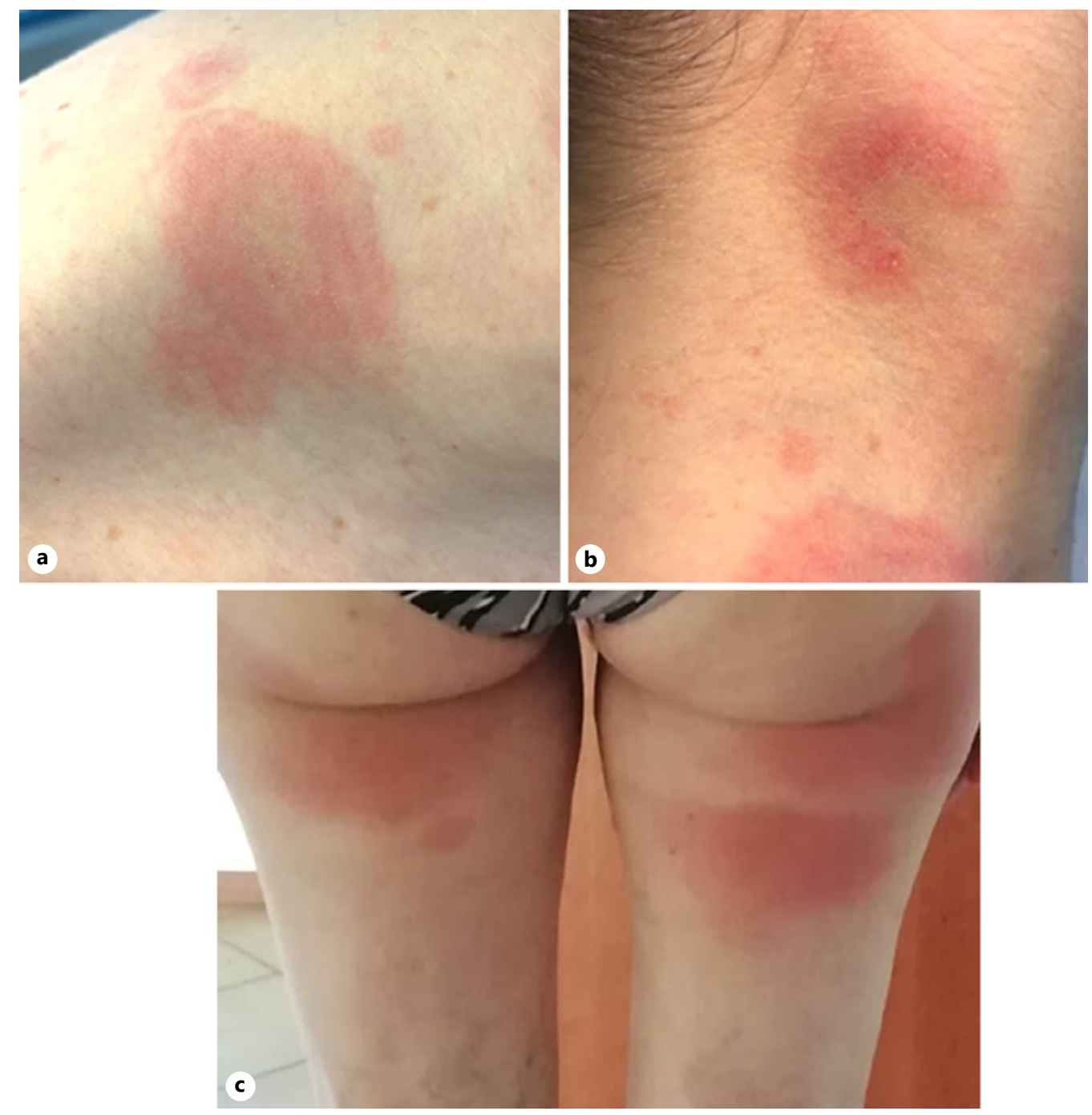

Fig. 1. a, b Annular plaques on the neck, presenting for 3 years always in spring (patient 3). c Erythematous annular plaques, localized bilaterally at the thighs for 3 consecutive years in summer (patient 4).

case 3 revealed a predominance of $\mathrm{T}$ cells with a small B-cell component. $\mathrm{T}$ cells were mixed CD4+ and CD8+ with preserved expression of CD7, CD5, and CD2 markers. Moreover, a moderate number of CD123+ plasmacytoid dendritic cells and CD1a+ dendritic cells were noted (Fig. 3).

\section{Discussion}

EAC is a rare disorder clinically characterized by the presence of annular erythematous lesions that enlarge centrifugally with central clearing $[1,2]$. A fine scale may be present inside the advancing edge. The lesions in most of cases tend to involve the extremities, in particular the thighs and legs, and less frequently the trunk $[1,2]$. The peculiarity of AR-EAC is the recurrence for years of these cutaneous manifestations, always in the same period of the year. In most cases, the recurrence was noted during summer [1-3]. Only 14 cases of AR-EAC have been described previously (Table 1). Including our cases, patients' age ranged 
Fig. 2. Inflammatory infiltrate extended to the deep dermis. No eosinophils were detected, similarly to the deep EAC type (patient 3; HE. $\times 8$ (a); HE. $\times 200$ (b)). HE, hematoxylin and eosin; EAC, erythema annulare centrifugum.

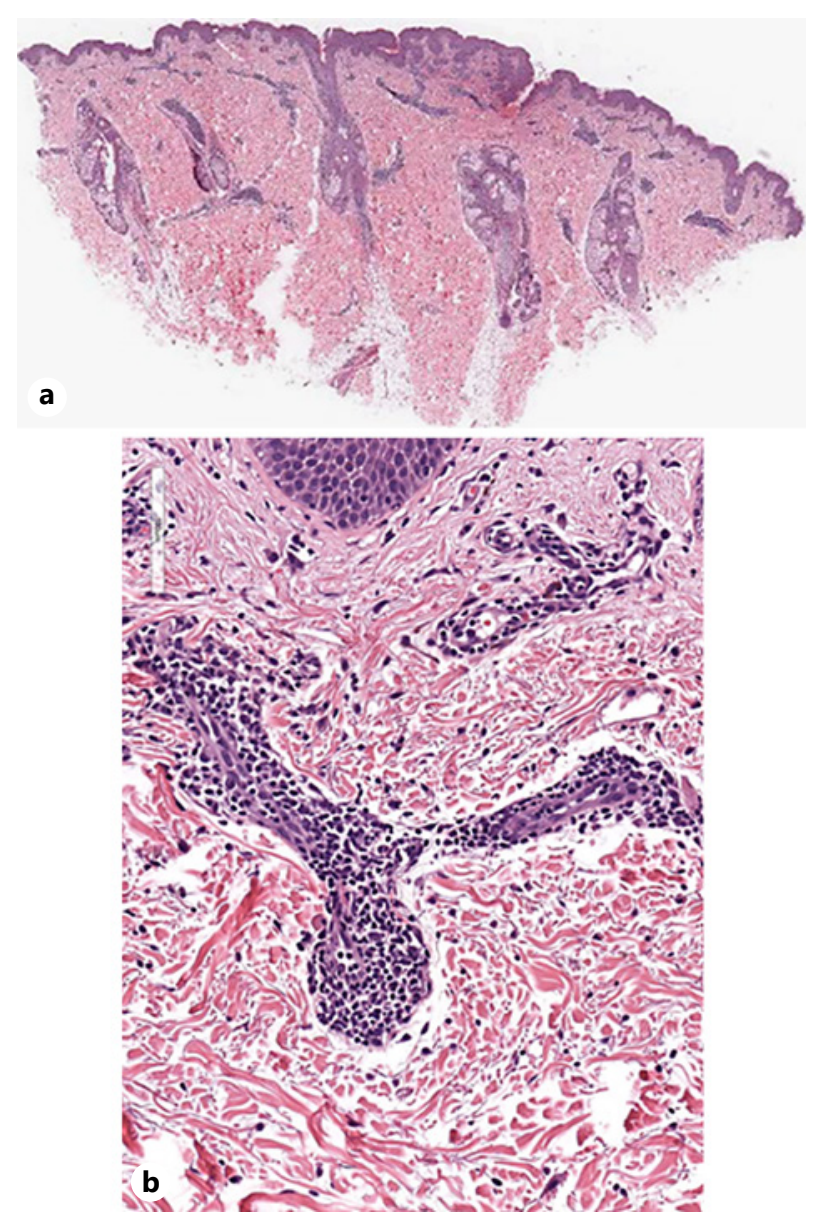

from 16 to 83 years, with a mean age of 47 years. In most cases, AR-EAC has been observed in women, with a female-to-male ratio of 2.4:1 [1-9]. Lesions were predominantly located at the extremities, and recurrences were more commonly in summer. The disease duration ranged from 1 to 30 years, with a median duration of 12 years. No associated diseases have been reported, and no obvious inducing factors (e.g., insect bites, drugs, and infections) have been disclosed. Interestingly, all cases reported are from Southern Europe and Japan, except of 1 from Chicago, IL, USA.

Histopathologically, AR-EAC is identical to EAC, and all cases described previously were of the superficial type. Our patient 3 is the first AR-EAC deep type. AR-EAC should be differentiated from the classical form of EAC and with other annular erythematous skin diseases, such as erythema chronicum migrans, erythema marginatum, urticaria, Jessner-Kanof disease, and cutaneous lupus erythematous $[3,10,11]$. Erythema papulatum centrifugum and palpable migratory arciform erythema are characterized by a single or multiple recurrent annular or semiannular erythematous lesions with a central regression; histopathologically, they are characterized by a superficial perivascular inflammation that may extend around the adnexa in the mid-dermis $[11,12]$.

The etiology and the pathogenesis of AR-EAC are not understood [6]. It has been hypothesized that AR-EAC is the result of a hypersensitive reaction to some external or internal causative factors, such as insect bite, infectious diseases, hormonal disturbances, drugs, and neoplasms $[1,2]$. However, none of these associations has been described in AR-EAC patients, in contrast to the classical EAC form. Indeed, all cases described having AR-EAC are idiopathic. Yoshikuni 


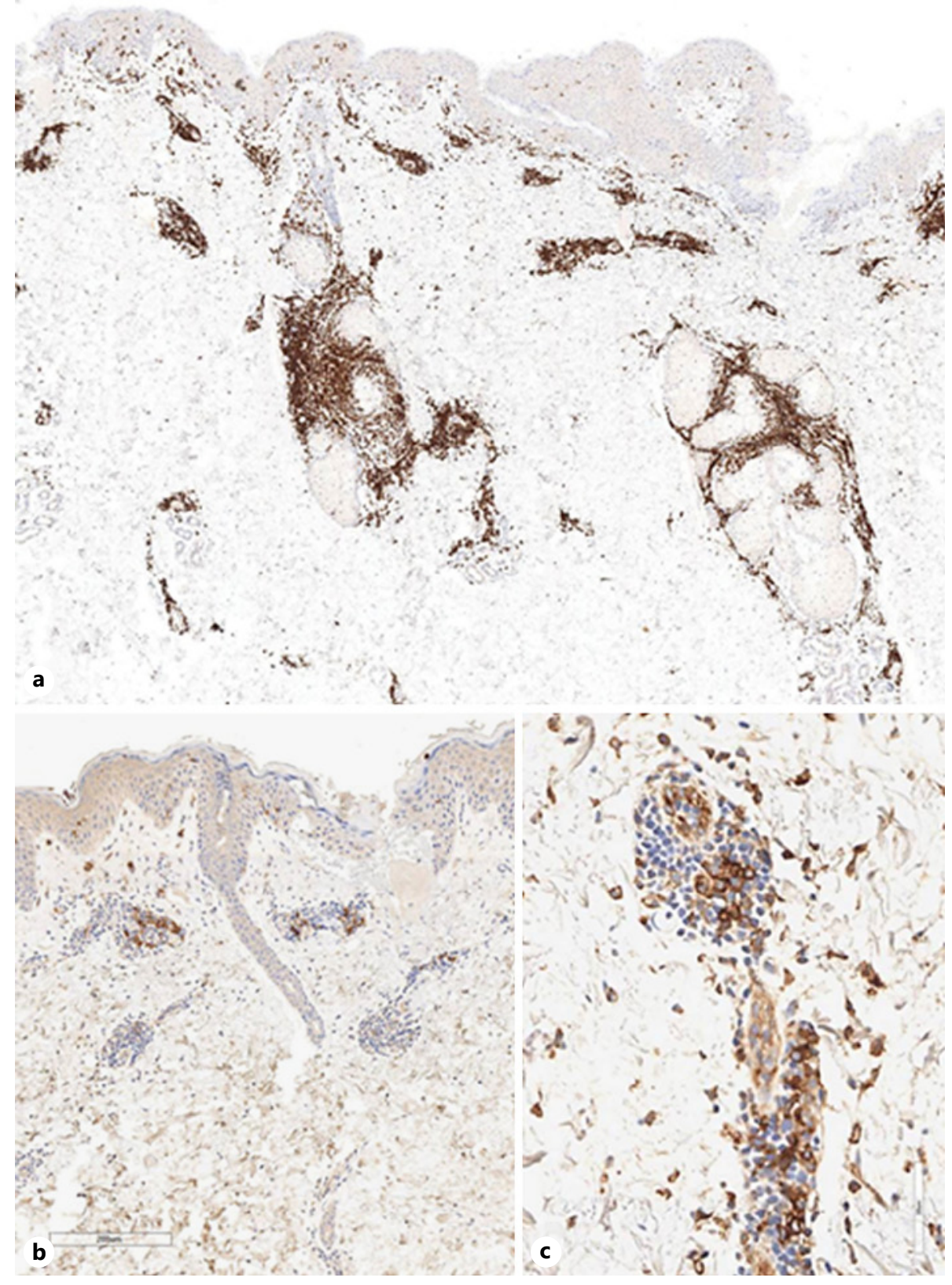

Fig. 3. Immunohistochemistry revealed T-cell $\left(\mathrm{CD}^{+}\right)$-dominated infiltrate $(\mathbf{a}, \times 40)$, with some $\mathrm{CD}_{1 \mathrm{a}^{+}}$ $(\mathbf{b}, \times 100)$ and $\mathrm{CD}_{123^{+}}(\mathbf{c}, \times 400)$ dendritic cells in the dermis.

et al. [7] first described the possible association between AR-EAC and hereditary lactate dehydrogenase M-subunit deficiency, characterized by the presence of muscle pains, easy fatigability, and the onset of myoglobinuria after an intense physical activity. Two are other such cases have been reported, who can be suspected based on extracutaneous symptoms [8,9]. AR-EAC is benign disorder, but it may recur for years with peculiar annually recurrences in the same time frame [2]. The lesions resolve spontaneously without leaving skin marks. It has been hypothesized that the typical periodical course of AR-EAC in summer may be explained by a photosensitivity reaction and the presence of environmental factors, such as temperature, seasonal plants, or fungus, and it is believed to be a sweating-related dermatitis [2]. However, in most cases, lesions are not located in sun-exposed areas. Although lesions were 
Table 1. Annually recurring erythema annulare centrifugum: review of the literature

\begin{tabular}{rlllcl}
\hline Case & $\begin{array}{l}\text { Gender/ } \\
\text { age }\end{array}$ & Localization & $\begin{array}{l}\text { Seasonal } \\
\text { recurrence }\end{array}$ & $\begin{array}{l}\text { Duration, Author } \\
\text { years }\end{array}$ & \\
\hline 1 & F/60 & Legs, elbows & Spring/summer & 7 & Piñol Aguadé et al. [5] \\
2 & F/43 & Legs, arms & Summer & 17 & Piñol Aguadé et al. [5] \\
3 & F/36 & Arms, back & - & 1 & Janss et al. [6] \\
4 & F/76 & Chest, back, arms, legs & Summer & 8 & García Muret et al. [1] \\
5 & M/83 & Internal face of extremities & Summer & 23 & García Muret et al. [1] \\
6 & M/55 & Legs, arms & Summer & 13 & García Muret et al. [1] \\
7 & F/55 & Legs, arms & Spring/summer & 15 & García Muret et al. [1] \\
8 & F/46 & Legs, arms & Summer & 12 & Mandel et al. [2] \\
9 & F/51 & Thigh & Spring & - & Nazzari and Crovato [8] \\
10 & F/27 & Thigh & Spring/summer & - & Betti et al. [9] \\
11 & M/70 & Trunk, upper limbs & Summer & 30 & Anedda et al. [3] \\
12 & $-/ 16$ & - & - & - & Yoshikuni et al. [7] \\
13 & - & - & - & - & Yoshikuni et al. [7] \\
14 & F/60 & Arms, chest, thighs & - & 3 & Mshrai et al. [4] \\
15 & M/55 & Thighs & Summer & 4 & Our case \\
16 & F/27 & Thigh & Winter & 3 & Our case \\
17 & F/32 & Neck & Spring & 3 & Our case \\
18 & F/34 & Thighs & Summer & 3 & Our case \\
19 & M/25 & Thighs & Summer & 3 & Our case \\
\hline & & & & & \\
\hline
\end{tabular}

expected to resolve spontaneously, the application of topical medium to high potency corticosteroids and a brief course of systemic corticosteroids led to a faster disappearance of the lesions.

\section{Statement of Ethics}

The study is exempt from local ethical committee approval. A written informed consent was obtained from the patients for publication of this case report and any accompanied images.

\section{Conflict of Interest Statement}

The authors declared no potential conflicts of interest with respect to the research, authorship, and/or publication of this article.

\section{Funding Sources}

The authors received no financial support for the research, authorship, and/or publication of this article.

\section{Author Contributions}

M.M. wrote the paper; P.G. was seeing the patients and supervised manuscript writing; C.C. was the pathologist, and G.G. supervised the study and manuscript preparation. 


\section{References}

1 García Muret MP, Pujol RM, Gimenez-Arnau AM, Barranco C, Gallardo F, Alomar A. A Annually recurring erythema annulare centrifugum: a distinct entity? J Am Acad Dermatol. 2006;54:1091-5.

2 Mandel VD, Ferrari B, Manfredini M, Giusti F, Pellacani G. Annually recurring erythema annulare centrifugum: a case report. J Med Case Rep. 2015;9:236.

3 Anedda J, Atzori L, Agosta D, Ferreli C, Atzori MG, Pilloni L, et al. Annually recurring acro-erythema. G Ital Dermatol Venereol. 2020.

4 Mshrai H, Fallatah B, Alwafi D, Babkoor D, Al Sufyani H, Al Hawsawi K. Erythema annulare centrifugum (EAC): a case report of annually recurring EAC. J Health Sci. 2016;6:74-6.

5 Piñol Aguadé J, Gimenez Camarasa J, De Moragas JM. Annually recurring erythema: an unusual variety of centrifugal annular erythema? Arch Argent Dermatol. 1966;16:75-87.

6 Janss G, Schmidt K, Gattuso P, Massa M, Welykyj S. An intensive care unit nurse with a recurring annular lesion. Erythema annulare centrifugum (EAC). Arch Dermatol. 1992;128:977-80.

7 Yoshikuni K, Tagami H, Yamada M, Sudo K, Kanno T. Erythematosquamous skin lesions in hereditary lactate dehydrogenase M-subunit deficiency. Arch Dermatol. 1986;122:1420-4.

8 Nazzari G, Crovato F. Annually recurring acroerythema and hereditary lactate dehydrogenase M-subunit deficiency. J Am Acad Dermatol. 1992;27:262-3.

9 Betti R, Gualandri L, Inselvini E, Crosti C. Annual recurrent annular acroerythema without lactate dehydrogenase M-subunit deficiency. J Eur Acad Dermatol Venereol. 1999;12:270-2.

10 McDaniel B, Cook C. Erythema annulare centrifugum. StatPearls [Internet]. Treasure Island, FL: StatPearls Publishing; 2020.

11 Song Z, Chen W, Zhong H, Ye Q, Hao F. Erythema papulosa semicircularis recidivans: a new reactive dermatitis? Dermatitis. 2012;23:44-7.

12 Wagner G, Bartsch S, Rose C, Sachse MM. Palpable arciforme migratory erythema. Hautarzt. 2012;63:965-8. 